\title{
Original Data
}

National Cancer Institute

\section{Source}

National Cancer Institute. Original Data. NCI Thesaurus. Code C142623.

The values reported in the initial documents used to record study data. 